\title{
Improved survival in metastatic breast cancer: results from a 20-year study involving 1033 women treated at a single comprehensive cancer center
}

\author{
Anja Welt ${ }^{1} \cdot$ Simon Bogner ${ }^{1} \cdot$ Marina Arendt $^{2,4} \cdot$ Josef Kossow $^{1,5} \cdot$ Antonia Huffziger $^{1,6} \cdot$ Christian Pohlkamp $^{1,7}$. \\ Heike Steiniger ${ }^{1,8}$. Ute Becker ${ }^{1,9} \cdot$ Ferras Alashkar $^{1,10}$. Marzena Kohl ${ }^{1,11}$. Marcel Wiesweg ${ }^{1} \cdot$ Heike Richly $^{1}$. \\ Jörg Hense ${ }^{1} \cdot$ Max E. Scheulen ${ }^{1,11} \cdot$ Martin Schuler $^{1,3} \cdot$ Siegfried Seeber $^{1,12} \cdot$ Mitra Tewes $^{1}$
}

Received: 1 February 2020 / Accepted: 12 March 2020 / Published online: 18 March 2020

(c) The Author(s) 2020

\begin{abstract}
Purpose Diagnosis and treatment of breast cancer have changed profoundly over the past 25 years. The outcome improved dramatically and was well quantified for early stage breast cancer (EBC). However, progress in the treatment of metastatic disease has been less convincingly demonstrated. We have studied survival data of patients with metastatic breast cancer (MBC) from a large academic cancer center over a period of 20 years.

Methods Data from 1033 consecutive MBC patients who were treated at the Department of Medical Oncology of the West German Cancer Center from January 1990 to December 2009 were retrospectively analyzed for overall survival (OS) and risk factors. Patients were grouped in 5-year cohorts, and survival parameters of each cohort were compared before and after adjustment for risk factors.

Results Overall survival of patients with MBC treated at specialized center has significantly improved from 1990 to 2010 (hazard ratio 0.7, 95\% CI 0.58-0.84). The increments in OS have become less profound over time (median OS 1990-1994: 24.2 months, 1995-1999: 29.6 months, 2000-2004: 36.5 months, 2005-2009: 37.8 months).

Conclusion Survival of patients with MBC has improved between 1990 and 2004, but less from 2005 to 2009. Either this suggests an unnoticed shift in the patient population, or a lesser impact of therapeutic innovations introduced in the most recent period.
\end{abstract}

Keywords Long-term survival $\cdot$ Metastatic breast cancer $\cdot$ New drugs

Anja Welt

anja.welt@uk-essen.de

1 Department of Medical Oncology, West German Cancer Center, University Hospital Essen, University DuisburgEssen, Hufelandstrasse 55, 45147 Essen, Germany

2 Institute for Medical Informatics, Biometry and Epidemiology, University Hospital Essen, University Duisburg-Essen, Essen, Germany

3 German Cancer Consortium (DKTK), Partner Site University Hospital Essen, Essen, Germany

4 Department of Computer Science, University of Applied Sciences and Arts, Dortmund, Germany

5 Department of Surgery, Herz-Jesu-Krankenhaus, Münster, Germany
6 Department of Medicine, Augusta Krankenhaus, Düsseldorf, Germany

7 Munich Leukemia Laboratory (MLL), München, Germany

8 Practice for Internal Medicine, Hematology and Oncology, Oberhausen, Germany

9 Department of Medicine I, St. Bernhard Hospital, Kamp-Lintfort, Germany

10 Department of Hematology, West German Cancer Center, University Hospital Essen, University Duisburg-Essen, Essen, Germany

11 Practice for General Medicine, Essen, Germany

12 Practice for Internal Medicine, Preventicum, Essen, Germany 


\section{Introduction}

Improved surgical, radiation and systemic therapies clearly have a positive impact on OS of women with EBC (EBCTCG 2015). However, it is still a matter of debate if survival of patients with MBC has equally improved. Since the 1990s, many new agents were introduced in the clinical care of MBC. These include taxanes, aromatase inhibitors, fulvestrant, capecitabin, vinorelbin, trastuzumab, liposomal doxorubicin, carboplatin, bevacizumab and lapatinib. Supportive therapy was optimized by recombinant granulopoiesis and erythropoiesis stimulating growth factors, and the morbidity of bone metastases was reduced by the use of bisphosphonates and denosumab. Recently, eribulin, everolimus, pertuzumab, trastuzumab emtansine (T-DM1), palbociclib, ribociclib and abemaciclib as well as atezolizumab, further expanded treatment options. This impressive therapeutic armamentarium heavily impinges on the OS outcome of randomized clinical trials conducted with new agents or combinations in earlier line treatment of $\mathrm{MBC}$, as multiple options for postprogression therapy are available.

Until 2018 only very few drugs were able to demonstrate an OS benefit in clinical trials of MBC: exemestane was superior to megestrol acetate, docetaxel was more active than mitomycin/vinblastine and docetaxel plus capecitabin were more effective than docetaxel alone. In HER2-positive MBC, chemotherapy plus trastuzumab led to better survival than chemotherapy alone. Docetaxel/ trastuzumab was further improved by the addition of pertuzumab and T-DM1 showed better overall survival compared to physician's choice treatment in heavily pretreated patients (Kaufmann et al. 2000; Nabholtz et al. 1999; O'Shaughnessy et al. 2002; Slamon et al. 2001; Baselga et al. 2012; Verma et al. 2012). Still, some of these few positive studies were criticized because of clearly suboptimal second and third line treatments following progression on study therapy (O'Shaughnessy et al. 2002; Feher et al. 2005).

Recently, a significant survival benefit for a new agent could be demonstrated in MBC: Patients with hormone receptor positive tumors had improved survival with endocrine therapy (ET) plus a CDK4/6 inhibitor compared to ET alone (Im et al. 2019; Slamon et al. 2019; Sledge et al. 2019), and nab-Paclitaxel (nab-P) plus Atezolizumab was shown to be superior to nab-P alone in a defined subgroup of patients with triple negative MBC (TNBC) (Schmid et al. 2018).

Most phase III studies leading to approval of new MBC drugs have relied on superiority in surrogate endpoints such as progression-free survival (PFS). Examples include bevacizumab, fulvestrant, aromatase inhibitors, everolimus, eribulin and CDK4/6 inhibitors. Assumable, this is explained by a dilution of the effect of the respective study drug on OS because of multiple effective options for next-line therapy.

Registries of comprehensive cancer centers (CCCs) may provide a useful resource for the analysis of trends of MBC mortality over extended periods, which correlate with the availability of new therapeutic options. While patient populations treated at CCCs may be positively selected, the high level of expertise and specialization of physicians and nurses, as well as the rapid access to diagnostic and therapeutic innovations assures high quality of care. Hence, relative changes in long-term outcomes that are obtained by a stable interdisciplinary team of MBC experts are well suited to detect the survival impact of therapeutic innovations that are introduced on specific periods. Examples for such analyses have been published from several major CCCs focusing on patients with secondary (Giordano et al. 2004; Chia et al. 2007; Sundquist et al. 2017) or de novo (Dawood et al. 2015; Ruiterkamp et al. 2011; Andre et al. 2004) MBC. These studies have demonstrated a stepwise improvement of OS over several decades. However, additional studies failed to demonstrate OS improvements for the entire population of MBC patients (Nakano et al. 2015; Tevaarwerk et al. 2013) or even suggested a trend for the deterioration of OS (Ufen et al. 2014; Hölzel et al. 2017).

Against this background, we conducted a long-term analysis of survival outcomes of women with MBC treated by the breast cancer team at the Department of Medical Oncology of the West German Cancer Center from 1990 to 2009.

\section{Patients and methods}

\section{Patient population}

The West German Cancer Center at University Hospital Essen is one of 13 Oncology Centers of Excellence designated by the Deutsche Krebshilfe (German Cancer Aid). Routine clinical data extracted from patient charts and the electronic hospital information system of 1033 consecutive MBC patients treated at the Department of Medical Oncology from 1990 to 2009 were retrospectively analysed for probability of overall survival (OS), 4-year survival (4YS) and 5-year survival rates (5YS). This population was grouped into four cohorts based on the year of first diagnosis of metastasis (cohort 1: 1990-1994, cohort 2: 1995-1999, cohort 3: 2000-2004 and cohort 4: 2005-2009). All data analyses were conducted in a pseudonymized way. The relevant ethics committee of the Medical Faculty of the University of Duisburg-Essen (No. 17-7608-BO) approved the study. 
Demographic data of patients were recorded including hormone receptor (HR) status, date of first diagnosis of breast cancer and of metastatic disease, localization of metastasis, and date of last contact or death. Particular attention was paid to high-risk patients with HR negative disease and/or visceral metastasis. HER2 status was mostly unknown in patients presenting in the years 1990-1999; accordingly, we decided to exclude HER2 status from this primary analysis.

The primary objective of the study was to evaluate the association between the period of diagnosis of MBC and survival.

\section{Statistics}

Statistics were calculated using IBM SPSS Statistics version 21.0 (Chicago Illinois, USA) and analyzed using SAS 9.4 (SAS Institute, Cary, North Carolina). Frequencies and percentages, means, medians and corresponding standard deviations were computed as well as 4-year, 5-year and median OS whenever applicable. Kaplan-Meier survival analysis was applied to estimate cumulative probabilities of OS. The log-rank test was used to compare survival rates with respect to date of initial diagnosis, diagnosis of $\mathrm{MBC}$, site of distant relapse, disease-free interval and hormone receptor status. Cox proportional hazard models were used to investigate the impact of year of diagnosis on all-cause mortality for the time intervals 1990-1994, 1995-1999, 2000-2004, and 2005-2009. As time to event, we used time to death beginning with the date of the first diagnosis of metastasis in months. We adjusted for site of metastasis, HR status and disease-free interval (DFI). $P$-values $<0.05$ were considered statistically significant.

\section{Results}

\section{Cohort characteristics}

In total, fully evaluable data could be retrieved for 1033 consecutive patients who were all included in this analysis. Survival data were reported as of April 2016. There were 933 deaths $(90.3 \%)$. The median follow-up period for surviving patients after the first diagnosis of MBC was 2.53 years. Cohort sizes were 279, 408, 143 and 203 patients for cohorts $1,2,3$ and 4. Patient characteristics of the entire population and the four cohorts are shown in Table 1 with $p$-values from a $Z$ test (percentage proportions) or $T$ test (absolute numbers) of each cohort against the whole population.

Patient characteristics were not evenly distributed across the four cohorts. The median age at the time of diagnosis of metastatic disease ranged from 52 to 56 years with significant differences. The proportion of patients aged $<50$ years, representing mostly patients with premenopausal status, decreased over time from cohort 1 (114 pts, $40.9 \%, p=0.97$ ), cohort 2 (193 pts, $47.3 \%, p=0.03)$, cohort $3(49,34.7 \%$, $p=0.11)$ to cohort $4(65,32 \%, p=0.02)$.

There was a clear time trend in the proportion of patients with unknown HR status: In the first cohort (1990-1994), nearly $22 \%$ of patients had unknown HR status. This fraction steadily decreased to $<1 \%$ in the most recent cohort (2005-2009). Consequently, the proportion of patients with HR positive disease was higher in cohorts $3(71 \%, p=0.01)$ and $4(67 \%, p=0.03)$ as compared to cohorts $1(53 \%)$ and $2(56 \%)$.

The fraction of patients with HR negative disease has a larger measurement error due to its smaller weight. Comparison of cohort 4 to cohort 1 , however, shows a proportional increase compatible with the hypothesis of stable underlying characteristics subject to more widespread testing.

The proportion of patients within each cohort who presented with $\mathrm{MBC}$ at initial diagnosis ranged from $9.8 \%$ (cohort 2) and $11.8 \%$ (cohort 1) to $16.1 \%$ (cohort 3) and $14.8 \%$ (cohort 4 ).

Testing the cohorts against the entire population reveals non-significant p-values well above 5\% (Table 3). A test of the largest difference, cohort 3 against cohort 2 , gives the marginal $p$-value of 0.053 .

For the complementary large fraction of patients with metastatic recurrence, the DFI was detailed for each cohort and showed a significant shift from early (DFI $<18$ months) to late recurrence (DFI $>36$ months). The more recent cohorts thus have a significant $(p<0.01)$ longer median DFI which increased from 25.9 to $27.6,38$ and 41 months in the cohorts 1, 2, 3 and 4, respectively.

No statistically significant differences were observed between the four cohorts with respect to the site of metastatic disease at the time of the first diagnosis of metastasis. Visceral metastasis was present in 54\% to $64 \%$ of patients with no relevant change over time. "Bone only" metastasis were seen in $17 \%$ to $24 \%$ of patients without a relevant change over time.

\section{Survival estimates and risk factors}

Overall survival from the first diagnosis of metastatic disease was compared between the four cohorts (Table 2). The median OS was 24.2 months, 29.6 months, 36.5 month and 37.8 months in patients who developed metastasis during 1990-1994, 1995-1999, 2000-2004 and 2005-2009, i.e. in cohorts 1,2, 3 and 4. Overall survival differed significantly between the four cohorts $(p<0.0001)$. 4YS rates were $22.6 \%, 29.7 \%, 36.4 \%$ and $37.4 \%, 5 \mathrm{YS}$ rates were $14 \%, 23 \%$, $30.8 \%$ and $24.6 \%$, respectively (see Table 2 ). The site of metastasis, HR status and DFI were identified as significant risk factors for OS (Table 2, bottom). 
Table 1 Characteristics of patients with metastatic breast cancer, stratified by time period of diagnosis of metastatic disease

\begin{tabular}{|c|c|c|c|c|c|}
\hline \multirow[t]{2}{*}{ Characteristic } & \multicolumn{5}{|c|}{ Years of recurrence: No of patients $(\%)$ [ $p$ value for test cohort against population] } \\
\hline & $\begin{array}{l}\text { 1990-2009 } \\
\text { All }\end{array}$ & $\begin{array}{l}\text { 1990-1994 } \\
\text { Cohort } 1\end{array}$ & $\begin{array}{l}1995-1999 \\
\text { Cohort } 2\end{array}$ & $\begin{array}{l}2000-2004 \\
\text { Cohort } 3\end{array}$ & $\begin{array}{l}2005-2009 \\
\text { Cohort } 4\end{array}$ \\
\hline$N(\%)$ & $1033(100 \%)$ & $279(27 \%)$ & $408(40 \%)$ & $143(13.8 \%)$ & $203(19.7 \%)$ \\
\hline FUP, median [months] & 30.3 & 24.2 & 29.6 & 36.5 & 37.8 \\
\hline \multicolumn{6}{|l|}{ Age at dissemination [years] } \\
\hline Median & 52.2 & $52[p=0.55]$ & $50.7[p<0.01]$ & $56.2[p<0.01]$ & $55.1[p<0.01]$ \\
\hline Range & $45.2-60$ & $44.8-59.9$ & $43.6-58$ & $47.9-62.2$ & $48-63.1$ \\
\hline$<50$ & $421(40.8 \%)$ & $114(40.9 \%)[p=0.97]$ & $193(47.3 \%)[p=0.03]$ & $49(34.3 \%)[p=0.11]$ & $65(32 \%)[p=0.02]$ \\
\hline$\geq 50$ & $612(59.2 \%)$ & $165(59.1 \%)$ & $215(52.7 \%)$ & $94(65.7 \%)$ & $138(68 \%)$ \\
\hline \multicolumn{6}{|l|}{ Hormone receptor $N(\%)$} \\
\hline Positive & $613(59.3 \%)$ & $148(53.1 \%)[p=0.07]$ & $229(56.1 \%)[p=0.30]$ & $101(70.6 \%)[p=0.01]$ & $135(66.5 \%)[p=0.03]$ \\
\hline Negative & $307(29.7 \%)$ & $70(25.1 \%)[p=0.10]$ & $136(33.3 \%)[p=0.27]$ & $34(23.8 \%)[p=0.13]$ & $67(33 \%)[p=0.40]$ \\
\hline Unknown & $113(10.9 \%)$ & $61(21.9 \%)[p<0.01]$ & $43(10.5 \%)[p=0.82]$ & $8(5.6 \%)[p=0.04]$ & $1(1 \%)[p<0.01]$ \\
\hline \multicolumn{6}{|l|}{ First metastasis $N(\%)$} \\
\hline $\begin{array}{l}\text { Metastasis as disease recur- } \\
\text { rence }\end{array}$ & $907(87.8 \%)$ & $246(88.2 \%)$ & $368(90.2 \%)$ & $120(83.9 \%)$ & $173(85.2 \%)$ \\
\hline $\begin{array}{l}\text { Metastasis at initial diagnosis } \\
\text { of breast cancer }\end{array}$ & $126(12.2 \%)$ & $33(11.8 \%)[p=0.89]$ & $40(9.8 \%)[p=0.20]$ & $23(16.1 \%)[p=0.20]$ & $30(14.8 \%)[p=0.31]$ \\
\hline \multicolumn{6}{|l|}{$\mathrm{DFI}^{*} N(\%)$} \\
\hline$<18$ months & $210(20.3 \%)$ & $70(25.1 \%)[p=0.08]$ & $96(23.5 \%)[p=0.18]$ & $17(11.9 \%)[p=0.02]$ & $27(13.3 \%)[p=0.02]$ \\
\hline $18-35$ months & $226(21.9 \%)$ & $66(23.7 \%)[p=0.47]$ & $99(24.3 \%)[p=0.35]$ & $26(18.2 \%)[p=0.31]$ & $35(17.2 \%)[p=00.13]$ \\
\hline$\geq 36$ months & $471(45.6 \%)$ & $110(39.4 \%)[p=0.06]$ & $173(42.4 \%)[p=0.27]$ & $77(53.9 \%)[p=0.07]$ & $111(54.7 \%)[p=0.02]$ \\
\hline DFI, median [months] & 30.9 & $25.9[p<0.01]$ & $27.6[p<0.01]$ & $38[p<0.01]$ & $41[p<0.01]$ \\
\hline \multicolumn{6}{|l|}{ Localization of first metastasis } \\
\hline Bone only & $215(20.8 \%)$ & $68(24.4 \%)[p=0.28]$ & $76(18.6 \%)[p=0.67]$ & $24(16.8 \%)[p=0.27]$ & $47(23.2 \%)[p=0.52]$ \\
\hline Liver and/or lung & $608(58.9 \%)$ & $152(54.5 \%)[p=0.13]$ & $261(64 \%)[p=0.08]$ & $83(58 \%)[p=0.81]$ & $112(55.2 \%)[p=0.29]$ \\
\hline Other & $210(20.3 \%)$ & $59(21.1 \%)[p=0.71]$ & $71(17.4 \%)[p=0.19]$ & $36(25.1 \%)[p=0.16]$ & $44(21.7 \%)[p=0.51]$ \\
\hline
\end{tabular}

Significant deviations of cohort characteristics versus the whole population are high-lighted in bold

DFI interval from first diagnosis of breast cancer to first diagnosis of metastasis, FUP interval from diagnosis of metastasis to last contact or death

Kaplan-Meier survival curves for OS for the four cohorts are shown in Fig. 1. Survival for cohort 2 was above that of cohort 1 , as was survival of cohort 3 relative to cohort 2 . The surviving fraction of the most recent cohort 4 hardly differs from its previous cohort 3 . The corresponding curves cross multiple times.

Table 3 shows the cohort-to-cohort comparison of OS. Data are given both for unadjusted survival (top) as well as after adjustment (bottom) for the risk factors "site of metastasis", "HR status" and "DFI", as identified in Table 2. Risk adjustment for relevant variables shall account for the differences in the distribution of risk factors between cohorts as shown in Table 2 and described above.

In the unadjusted analysis, we find a significant increase of OS from cohort 1 to cohort 2 (HR $0.78[0.66 ; 0.90]$ ) and again, slightly smaller, from cohort 2 to cohort 3 (HR 0.81 $[0.67 ; 0.99])$. Cohort 4 , by contrast, does not differ from cohort 3 (HR $1.1[0.89 ; 1.39]$ ). After risk-adjustment, this picture remains intact with a further shift of the weight of gain in OS towards earlier years (cohort 1 to cohort 2: HR 0.71 , significant; cohort 2 to 3 : HR 0.92 , overlapping with unity; cohort 3 to 4: HR 1.07, overlapping with unity).

Comparison of the most recent cohort 4 to the most remote cohort 1 shows a stable and significant long-term improvement in OS (unadjusted HR 0.70 [0.58; 0.84]); riskadjusted HR $0.70[0.58 ; 0.85])$.

\section{Discussion}

In the current data set, we have observed an effect of year of diagnosis of MBC on OS. This is in line with findings from other high volume and expertise centers demonstrating a modest but significant increase in survival over time among patients with MBC. Giordano et al. published a seminal study of patients treated at the MD Anderson Cancer 
Table 2 Unadjusted outcome and risk factors

\begin{tabular}{|c|c|c|c|c|c|}
\hline Group of patients & $N$ & $\begin{array}{l}\text { OS, } \\
\text { median } \\
\text { [months] }\end{array}$ & 4YS [\%] & $5 \mathrm{YS}[\%]$ & $\begin{array}{l}\text { Logrank } \\
p \text {-value }\end{array}$ \\
\hline Total & 1033 & 30.3 & 30.4 & 22 & \\
\hline 1990-1994 & 279 & 24.2 & 22.6 & 14 & $<0.0001$ \\
\hline 1995-1999 & 408 & 29.6 & 29.7 & 23 & \\
\hline 2000-2004 & 143 & 36.5 & 36.4 & 30.8 & \\
\hline 2005-2009 & 203 & 37.8 & 37.4 & 24.6 & \\
\hline Bone only & 215 & 44.8 & 48.8 & 36.7 & $<0.0001$ \\
\hline $\begin{array}{l}\text { Liver and/or } \\
\text { lung }\end{array}$ & 608 & 24.7 & 23.5 & 16.5 & \\
\hline Other & 210 & 31.8 & 29.1 & 21 & \\
\hline $\mathrm{HR}+$ & 613 & 37.3 & 36.4 & 25.9 & $<0.0001$ \\
\hline $\mathrm{HR}-$ & 307 & 19.7 & 14.3 & 10.1 & \\
\hline HR unknown & 113 & 31.1 & 37.2 & 29.2 & \\
\hline Initial metastasis & 126 & 38.6 & 38.1 & 29.4 & $<0.0001$ \\
\hline DFI $<18$ month & 210 & 18.4 & 17.1 & 11.4 & \\
\hline $\begin{array}{l}\text { DFI } \\
18-35 \text { month }\end{array}$ & 226 & 25.4 & 21.7 & 15 & \\
\hline DFI $>35$ month & 471 & 37.5 & 37.4 & 27.2 & \\
\hline Age $<50$ years & 421 & 29.6 & 28.7 & 20.9 & 0.6087 \\
\hline Age $\geq 50$ years & 612 & 31.6 & 30.7 & 22.1 & \\
\hline
\end{tabular}

Median overall survival, probability of 4-year and 5-year overall survival of patients with metastatic breast cancer, according to time period of diagnosis of metastatic disease and according to prognostic factors

OS overall survival. 4YS, 4-year overall survival. 5YS 5-year overall survival
Center. Survival of 834 patients with recurrent breast cancer improved from 1974 to 2000 with $1 \%$ reduction in the risk of death for each successive year (2003). A study group from British Columbia found a relative prolongation of approximately $30 \%$ in OS for 2.150 patients with MBC diagnosed between 1991 and 2001 (Chia et al. 2007). A Swedish study group demonstrated improved survival from 13 to 33 months and 5 YS from 10 to $27 \%$ for 784 patients diagnosed with MBC from 1985 to 2014 (Sundquist et al. 2017). However, not all of the prior analyses were adjusted for DFI and other covariates. Several major CCCs focused on patients with de novo MBC also reporting an improvement of OS for the entire population (Ruiterkamp et al. 2011; Dawood et al. 2015; Andre et al. 2004).

There is evidence that the most striking effect is given in the HER2 positive subset (Tevaarwerk et al. 2013; Nakano et al. 2015; Sundquist et al. 2017), partly represented as "HR negative disease" because information regarding HER2 status was missing in some analyses (Tevaarwerk et al. 2013). Clearly, this subgroup of patients has shown an OS benefit obviously caused by the introduction of HER2-targeted therapies (Slamon et al. 2001; Dawood et al. 2010; Baselga et al. 2012).

In general, more patients were treated with systemic therapies in the neo-/adjuvant setting since the 1990s. This is consistent with the international trend of decline in breast cancer mortality in western countries (DeSantis et al. 2014; Carioli et al. 2017) which cannot only be

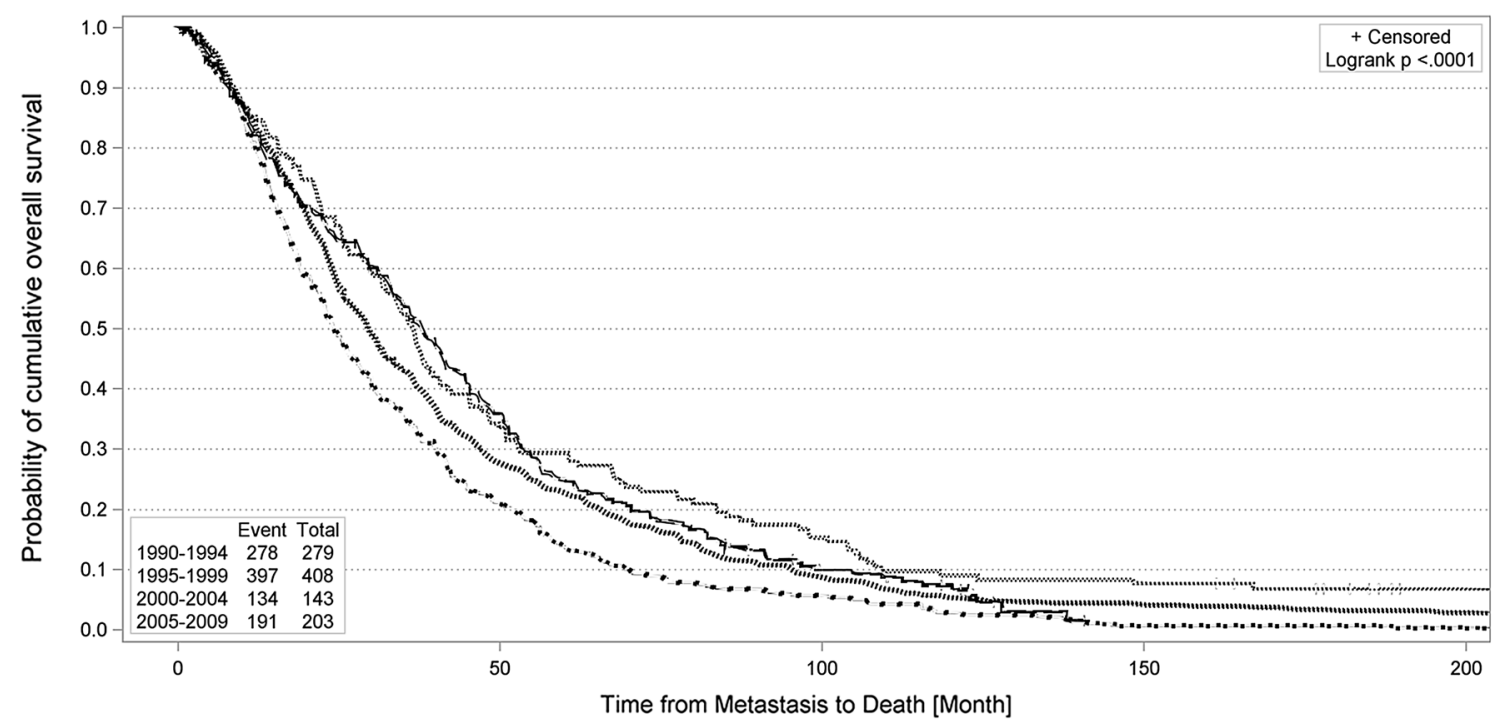

\begin{tabular}{|c|c|c|c|c|c|c|c|c|c|c|c|c|c|c|c|c|c|c|c|c|c|}
\hline \multirow[b]{2}{*}{$1990-1994$} & \multirow[b]{2}{*}{279} & \multirow[b]{2}{*}{239} & \multirow[b]{2}{*}{164} & \multirow[b]{2}{*}{116} & \multicolumn{3}{|c|}{......... 1990-1994 } & \multicolumn{3}{|c|}{ 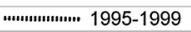 } & \multicolumn{3}{|c|}{ 2000-2004 ستسחسس } & \multicolumn{3}{|c|}{$---2005-2009$} & \multirow[b]{2}{*}{2} & \multirow[b]{2}{*}{2} & \multirow[b]{2}{*}{2} & \multirow[b]{2}{*}{1} & \multirow[b]{2}{*}{1} \\
\hline & & & & & 84 & 59 & 38 & 28 & 22 & 19 & 16 & 12 & 8 & 7 & 4 & 2 & & & & & \\
\hline $1995-1999$ & 408 & 357 & 281 & 200 & 151 & 113 & 93 & 72 & 59 & 44 & 36 & 28 & 22 & 19 & 18 & 17 & 16 & 15 & 13 & 13 & 12 \\
\hline 2000-2004 & 143 & 124 & 107 & 85 & 60 & 48 & 42 & 34 & 30 & 25 & 22 & 14 & 13 & 12 & 12 & 11 & 11 & 8 & 6 & 1 & 1 \\
\hline 2005-2009 & 203 & 176 & 143 & 122 & 95 & 73 & 50 & 42 & 34 & 25 & 17 & 14 & 10 & 2 & 1 & 0 & & & & & \\
\hline
\end{tabular}

Fig. 1 Probability of overall survival of patients with metastatic breast cancer stratified by time period of diagnosis of metastatic disease using Kaplan-Meier Analysis 
Table 3 Overall survival comparison cohort-to-cohort over time, before and after risk adjustment

\begin{tabular}{|c|c|c|c|c|}
\hline cohort & 1990-1994 & 1995-1999 & 2000-2004 & 2005-2009 \\
\hline \multicolumn{5}{|c|}{$\begin{array}{l}\text { Incremental change in OS over time, hazard ratios, unadjusted; HR given for column compared to row } \\
(<1 \text { : better); }\end{array}$} \\
\hline 1990-1994 & 1 (Reference) & $0.78(0.66 ; 0.90)$ & $0.63(0.51 ; 0.77)$ & $0.70(0.58 ; 0.84)$ \\
\hline 1995-1999 & $1.29(1.11 ; 1.51)$ & 1 (Reference) & $0.81(0.67 ; 0.99)$ & $0.90(0.76 ; 1.07)$ \\
\hline 2000-2004 & $1.59(1.29 ; 1.96)$ & $1.23(1.01 ; 1.50)$ & 1 (Reference) & $1.11(0.89 ; 1.39)$ \\
\hline 2005-2009 & $1.43(1.19 ; 1.73)$ & $1.11(0.94 ; 1.32)$ & $0.90(0.72 ; 1.13)$ & 1 (Reference) \\
\hline \multicolumn{5}{|c|}{$\begin{array}{l}\text { Incremental change in OS over time, hazard ratios adjusted for risk factors "site of metastasis", "HR } \\
\text { status" and "DFI until metastasis"; HR ratios given for column compared to row ( }<1 \text { : better) }\end{array}$} \\
\hline 1990-1994 & 1 (Reference) & $0.71(0.61 ; 0.84)$ & $0.66(0.53 ; 0.82)$ & $0.70(0.58 ; 0.85)$ \\
\hline 1995-1999 & $1.40(1.20 ; 1.64)$ & 1 (Reference) & $0.92(0.76 ; 1.18)$ & $0.99(0.83 ; 1.18)$ \\
\hline 2000-2004 & $1.52(1.23 ; 1.88)$ & $1.08(0.89 ; 1.33)$ & 1 (Reference) & $1.07(0.86 ; 1.34)$ \\
\hline $2005-2009$ & $1.53(1.27 ; 1.85)$ & $1.06(0.98 ; 1.27)$ & $0.91(0.73 ; 1.13)$ & 1 (Reference) \\
\hline
\end{tabular}

Most important results in incremental change in OS over time are high-lighted in bold contributed to the implementation of mammographic screening in the more recent years. On the other hand, the extensive use of systemic treatment in the neo-/adjuvant setting in recent years could have reduced the effectiveness of palliative systemic therapy in these groups, because a greater proportion of patients were no longer naïve for chemotherapy and endocrine treatment. Ufen et al. studied 1635 patients treated for MBC in three German cancer centers between 1980 and 2009. They failed to find an improvement but observed a decline of median OS over time which was associated with a shift towards more aggressive disease (2014). This corresponds with Hölzel et al. reporting a clear improvement of 5-year-survival in EBC over three decades, presumably caused by better perioperative systemic treatment leading to an extension of DFI. Paradoxically, this effect was accompanied by a reduction of median OS in patients with MBC (2017).

We here present 1033 consecutive patients with MBC treated between 1990 and 2009 at a major German comprehensive cancer center. No predefined sequence of treatment for metastatic breast cancer was applied. Treatment sequence was individualized as a function of prior treatment, tumor biology, the dynamics of metastasis, and patient preferences (particularly in relation to toxicities and treatment intervals) by a stable expert team.

Cohort 2 (1995-1999) was much larger than other cohorts. During this period our cancer center offered highdose chemotherapy with autologous stem cell transplantation in clinical studies as part of the therapeutic concept (Bojko et al. 2004). This is probably the main reason why more patients were assigned to our clinic.

Patient characteristics were unequally distributed across the four cohorts regarding HR status, age and DFI. The growing proportion of patients with known HR positivity may rather be explained by the adoption of routine testing of HR expression than by a real change in the distribution of HR positivity over time.

The significant increase in age over time may partially reflect the longer DFI between diagnosis and treatment of the primary breast tumor and metastasis. This is consistent with well-recognized improvements of multimodal treatment of primary breast cancer. More generally, this trend of rising age may indicate that the increase in life expectancy at birth for the overall population is not lost on women with MBC.

We find significant and relevant improvement in OS from 24.2 months to 38.8 months over this period of two decades. Our analyses with respect to the time of presentation (4 cohorts of distinct 5-year periods) and established risk factors (age, HR status, DFI, site of metastasis) showed that the gain in OS slowed over time. After risk adjustment, accounting for fluctuations over time in the patient characteristics, a significant gain is shown for the years 1995-1999 when compared to $1990-1995$, and a numerical, statistically nonsignificant but plausible gain for the period of 2000-2004 compared to 1995-1999. No further improvement is seen when cohort 4 (2005-2009) is compared to the immediately preceding cohort. However, the improvement in OS accrued in the first 15 years of the study sustains.

In agreement with Hölzel et al. (2017) our analyses showed a sustained increase of DFI over time from 25.9 (1990-1994) to 41.0 months (2005-2009), plausibly due to improvements in the primary treatment. Assuming the recurrent population is composed of more negatively selected cancer biologies, this could explain that increments in OS have become less profound over time despite of the introduction of new therapeutic agents in the treatment of MBC in Germany (1990-1994: paclitaxel; 1995-1999: docetaxel, letrozole, anastrozole; 2000-2004: exemestane, capecitabin, trastuzumab, fulvestrant, pegylated liposomal doxorubicin, gemcitabin; 2005-2009: bevacizumab, liposomal doxorubicin, lapatinib, nab-P). 
We recognize that the current study has limitations. Some degree of bias may be attributed to the retrospective character of this analysis. Additionally, HER2 status was not included in our evaluation, because it was unknown in many patients treated for MBC in the nineties. Therefore a subsequent analysis regarding HER 2 status of patients in cohorts 3 and 4 is planned soon. This will include a more recent cohort (2010-2014), too.

The strengths of this study include a large unselected patient population and extensive median follow-up time (median years: 2.53; range 1.30-4.49). OS results of the cohorts 3 (2000-2004; OS 36.5 months) and 4 (2005-2009; OS 37.8 months) are well in line with those reported from the prospective German TMK cohort study, namely median OS of 33.8 months reporting 1395 "real world" patients treated from 2007 to 2015 in various oncology practices and community hospitals in Germany (Fietz et al. 2017).

In sum, we report survival data of patients with MBC treated at the Department of Medical Oncology at the West German Cancer Center that show large improvements between 1990 and 2009. During this time, multiple effective anticancer agents that can palliate MBC have been introduced into routine practice. The rate of improvement, however, appears to have been slowing during the observation time of our study. This suggests either a negative selection of patients with recurrent breast cancer due to more effective perioperative therapy over time in EBC or a lesser impact of therapeutic innovations introduced in the most recent period. Since 2010, again many drugs were released in the treatment of MBC (eribulin, T-DM1, pertuzumab, everolimus, ribociclib, palbociclib, abemaciclib, atezolizumab) which hopefully will further improve survival with MBC.

Acknowledgements Open Access funding provided by Projekt DEAL.

\section{Compliance with ethical standards}

Conflict of interest AW: Novartis (consulting fees and grant), Pfizer, Amgen, Tesaro, Roche, AstraZeneca, Interplan (consulting fees); MW: Amgen (travel support), Roche (travel support); MS: Consultant (compensated)—AstraZeneca, Boehringer Ingelheim, Bristol-Myers Squibb, Novartis, Roche, Takeda: Stock ownership-none: Honoraries for CME presentations-Abbvie, Boehringer Ingelheim, BristolMyers Squibb, Lilly, MSD, Novartis, Pierre Fabre: Research funding to institution-AstraZeneca, Boehringer Ingelheim, Bristol MyersSquibb, Novartis; MA, SB, JK, AH, CP, HS, UB, FA, MK, HR, JH, MES, MT, SS declares that they have no conflict of interest.

Open Access This article is licensed under a Creative Commons Attribution 4.0 International License, which permits use, sharing, adaptation, distribution and reproduction in any medium or format, as long as you give appropriate credit to the original author(s) and the source, provide a link to the Creative Commons licence, and indicate if changes were made. The images or other third party material in this article are included in the article's Creative Commons licence, unless indicated otherwise in a credit line to the material. If material is not included in the article's Creative Commons licence and your intended use is not permitted by statutory regulation or exceeds the permitted use, you will need to obtain permission directly from the copyright holder. To view a copy of this licence, visit http://creativecommons.org/licenses/by/4.0/.

\section{References}

Andre F, Slimane K, Bachelot T et al (2004) Breast cancer with synchronous metastases: trends in survival during a 14-year period. J Clin Oncol 15:3302-3308

Baselga J, Cortés J, Kim SBN et al (2012) Pertuzumab plus trastuzumab plus docetaxel for metastatic breast cancer. New Engl $\mathbf{J}$ Med 366:109-119

Bojko P, Welt A, Schleucher R, Borquez D, Scheulen ME, Vanhoefer U, Poettgen C, Stuschke M, Broelsch CE, Stamatis G, Wilke H, Seeber S, Harstrick A (2004) High-dose chemotherapy with autologous stem cell transplantation in patients with oligometastatic breast cancer. Bone Marrow Transplant 34:637-643

Carioli G, Malvezzi M, Rodriguez T, Bertuccio P, Negri E, La Vecchia C (2017) Trends and predictions to 2020 in breast cancer mortality in Europe. Breast 36:89-95

Chia SK, Speers CH, Dyachkova Y, Kang A, Malfair-Taylor S, Barnett J, Coldman A, Gelmo KA, OReill Olivotto SEIA (2007) The impact of new chemotherapeutic and hormone agents on survival in a population-based cohort of women with metastatic breast cancer. Cancer 110:973-979

Dawood S, Broglio K, Buzdar AU, Hortobagyi GN, Giordano SH (2010) Prognosis of women with metastatic breast cancer by HER2 status and trastuzumab treatment: an institutional-based review. J Clin Oncol 28:92-98

Dawood S, Haaland B, Albarracin C (2015) Is the proportion of patients diagnosed with synchronous stage IV breast cancer who survive more than 2 years increasing over time? Oncology 89:79-87

DeSantis C, Ma J, Bryan L, Jemal A (2014) Breast Cancer statistics 2013. CA Cancer J Clin 64:52-62

Early Breast Cancer Trialists' Collaborative Group (EBCTCG) (2015) Aromatase inhibitors versus tamoxifen in early breast cancer: patient-level meta-analysis of the -randomised trials. Lancet 386:1341-1352

Feher O, Vodvarka P, Jassem J et al (2005) First-line gemcitabine versus epirubicin in postmenopausal women aged 60 or older with metastatic breast cancer: a multicenter, randomized, phase III study. Ann Oncol 16:988

Fietz T, Tesch H, Rauh J et al (2017) Palliative systemic therapy and overall survival of 1,395 patients with advanced breast cancer Results from the prospective German TMK cohort study. Breast 34:122-130

Giordano SH, Buzdar AU, Smith TL et al (2004) Is breast cancer survival improving? Cancer 100:44-52

Hölzel D, Eckel R, Bauernfeind F et al (2017) Improved systemic treatment for early breast cancer improves cure rates, modifies metastatic pattern and shortens post-metastatic survival: 35-year results from the Munich Cancer Registry. Cancer Res Clin Oncol 143:1701-1712

Im SA, Lu YS, Bardia A et al (2019) Overall Survival with Ribociclib plus Endocrine Therapy in Breast Cancer. N Engl J Med 381:307-316

Kaufmann M, Bajetta E, Dirix LY et al (2000) Exemestane is superior to megestrol acetate after tamoxifen failure in postmenopausal women with advanced breast cancer: results of a phase III randomized double-blind trial. The Exemestane Study Group. J Clin Oncol 18:1399-1411 
Nabholtz JM, Senn HJ, Bezwoda WR et al (1999) Prospective randomized trial of docetaxel versus mitomycin plus vinblastine in patients with metastatic breast cancer progressing despite previous anthracycline-containing chemotherapy. 304 Study Group. J Clin Oncol 17:1413-1424

Nakano M, Fujisue M, Tashima R et al (2015) Survival time according to the year of recurrence and subtype in recurrent breast cancer. Breast 24:588-593

O'Shaughnessy J, Miles D, Vukelja S et al (2002) Superior survival with capecitabine plus docetaxel comination therapy in anthracycline-pretreated patients with advanced breast cancer. J Clin Oncol 20:2812-2823

Ruiterkamp J, Ernst MF, de Munck L et al (2011) Improved survival of patients with primary distant metastatic breast cancer in the period of 1995-2008. A nationwide population-based study in the Netherlands. Breast Cancer Res Treat 128:495-503

Schmid P, Adams S, Rugo HS et al (2018) Atezolizumab and NabPaclitaxel in Advanced Triple-Negative Breast Cancer. N Engl J Med 379:2108-2121

Slamon DJ, Leyland-Jones B, Shak S et al (2001) Use of chemotherapy plus a monoclonal antibody against HER2 for metastatic breast cancer that overexpresses HER2. N Engl J Med 344:783-792

Slamon D, Neven P, Chia S et al (2019) Overall Survival with Ribociclib plus Fulvestrant in advanced breast cancer. N Engl J Med. https://doi.org/10.1056/NEJMoa1911149
Sledge GW Jr, Toi M, Neven P et al (2019) The Effect of Abemaciclib Plus Fulvestrant on overall survival in hormone receptor-positive, ERBB2-negative breast cancer that progressed on endocrine therapy-MONARCH 2: a randomized clinical trial. JAMA Oncol. https://doi.org/10.1001/jamaoncol.2019.4782

Sundquist M, Brudin L, Tejler G (2017) Improved survival in metastatic breast cancer 1985-2016. Breast 31:46-50

Tevaarwerk AJ, Gray RJ, Schneider BP et al (2013) Survival in Patients With Metastatic Recurrent Breast Cancer After Adjuvant Chemotherapy. Little Evidence of Improvement Over the Past 30 Years. Cancer 119:1140-1148

Ufen MP, Köhne CH, Wischneswky M et al (2014) Metastatic breast cancer: are we treating the same patients as in the past? Ann Oncol 25:95-100

Verma S, Miles D, Gianni L et al (2012) Trastuzumab Emtansine for HER2-positive advanced breast cancer. N Engl J Med 367:1783-1791

Publisher's Note Springer Nature remains neutral with regard to jurisdictional claims in published maps and institutional affiliations. 